\title{
Head-Media Interaction in Magnetic Recording
}

Avner Friedman

and

J. Ignacio Tello

The tape head interaction in magnetic recording is modeled by a coupled system of a second-order differential equation for the pressure and a fourth-order differential equation for the tape deflection. There is also the constraint that the spacing between the head and the tape remains positive. In this paper, we study the stationary one-dimensional case and establish the existence of a smooth solution.

\section{THE MODEL}

Figure 1 shows the magnetic tape modeled by $\hat{y}=\hat{u}(\hat{x}), 0<\hat{x}<\hat{L}$, and the magnetic head profile $\hat{y}=\hat{\delta}(\hat{x}), \hat{L}_{1} \leqslant \hat{x} \leqslant \hat{L}_{2}$. The spacing between the head and the tape is denoted by $\hat{h}(\hat{x})$, i.e.,

$$
\hat{h}(\hat{x})=\hat{u}(\hat{x})-\hat{\delta}(\hat{x}), \quad \hat{h}(\hat{x})>0, \quad \hat{L}_{1} \leqslant \hat{x} \leqslant \hat{L}_{2} .
$$

The tape is driven with velocity $V$, and its motion entrains air in the space between the head and the tape, with pressure $\hat{p}(\hat{x}), \hat{L}_{1} \leqslant \hat{x} \leqslant \hat{L}_{2}$. At the endpoints $\hat{x}=\hat{L}_{1}, \hat{x}=\hat{L}_{2}$, the pressure is equal to the atmospheric pressure.

After nondimensionalization, one obtains the following system for the tape $y=u(x)$ and the pressure $p(x)[1 ; 3$, Chap. 6],

$$
\begin{aligned}
\frac{\partial(p h)}{\partial x}-\varepsilon \frac{\partial}{\partial x}\left(\alpha h^{2} \frac{\partial p}{\partial x}+\beta h^{3} p \frac{\partial p}{\partial x}\right) & =0, \quad L_{1}<x<L_{2} \\
-\frac{\partial^{2} u}{\partial x^{2}}+\mu \frac{\partial^{4} u}{\partial x^{4}} & =K(p-1) \chi_{\left[L_{1}, L_{2}\right]}, \quad 0<x<L \\
u(x) & =h(x)+\delta(x), \quad h(x)>0 \quad \text { if } L_{1} \leqslant x \leqslant L_{2}
\end{aligned}
$$




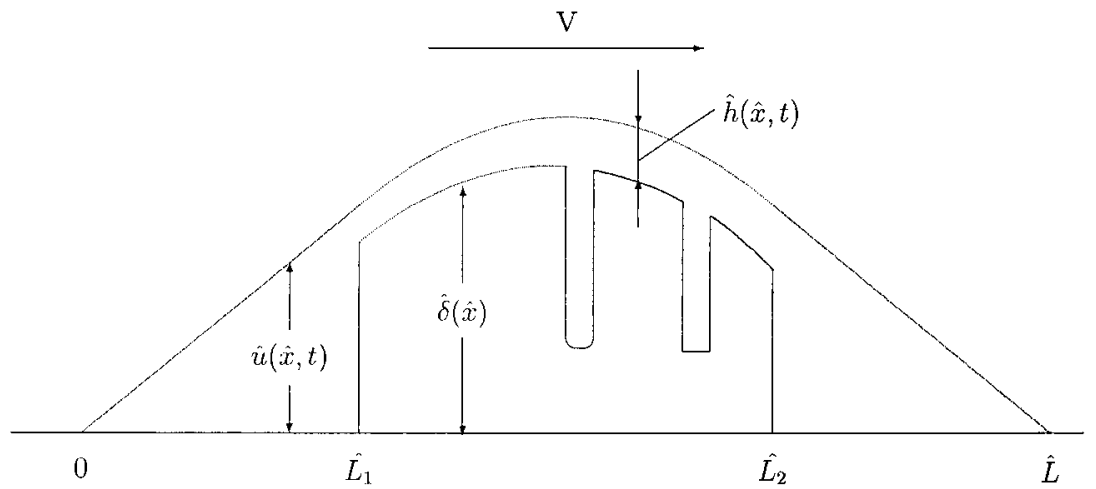

FIGURE 1

where $0<L_{1}<L_{2}<L, \chi_{\left[L_{1}, L_{2}\right]}$ is the characteristic function of the interval $\left[L_{1}, L_{2}\right]$, and, typically,

$$
\alpha \sim \frac{1}{10}, \beta \sim 1, L_{2}-L_{1} \sim 1, L \sim 10, K \sim 10^{4}, \varepsilon \sim 10^{-2} \text {, and } \mu \sim 10^{-3} ;
$$

$L_{1}$ and $L_{2}$ lie near of the middle of the interval $(0, L)$.

The boundary conditions are

$$
\begin{gathered}
p\left(L_{1}\right)=p\left(L_{2}\right)=1, \\
u=\frac{\partial u}{\partial x}=0 \quad \text { at } \quad x=0 \quad \text { and } \quad x=L .
\end{gathered}
$$

We assume throughout the paper that

$\delta$ is piecewise continuous with jump discontinuous at $\xi_{1}, \xi_{2}, \ldots, \xi_{s}$ where $\xi_{0}=L_{1}<\xi_{1}<\cdots<\xi_{s}<L_{2}=\xi_{s+1}$, and $\delta \in C^{1}\left[\xi_{i}, \xi_{i+1}\right]$ for $0 \leqslant i \leqslant s$,

and

$$
\delta\left(L_{1}\right)<\delta^{\prime}\left(L_{1}\right) L_{1}, \quad \delta\left(L_{2}\right)<\left(L_{2}-L\right) \delta^{\prime}\left(L_{2}\right) ;
$$

the case where $\delta(x)$ has no discontinuities may be considered as a special case of (1.6) (with $s=0$ ).

Note that the inequality $\delta\left(L_{1}\right)<\delta^{\prime}\left(L_{1}\right) L_{1}$ means that the tangent to the head at $x=L_{1}$ intersects the $x$-axis in the interval $\left(0, L_{1}\right)$. Similarly, the second inequality in (1.7) means that the tangent to the head at $x=L_{2}$ intersects the $x$-axis in the interval $\left(L_{2}, L\right)$.

Note also that $\varepsilon$ and $\mu$ are small numbers. In this paper we prove: 
THEOREM 1.1. Assume that (1.6) and (1.7) are satisfied. Then there exist positive constants $\varepsilon_{*}, \mu_{*}$ such that if $0<\varepsilon<\varepsilon_{*}, 0<\mu<\mu_{*}$, then the system (1.1)-(1.5) has a solution with $p \in W^{1, \infty}\left[L_{1}, L_{2}\right], u \in W^{4, \infty}[0, L]$, and $p>0, h>0$ in $\left[L_{1}, L_{2}\right]$.

Theorem 1.1 was proved in [4] under the assumption that

$$
\delta \in C^{2} \quad \text { and } \quad \delta^{\prime \prime}(x)<0, \quad L_{1} \leqslant x \leqslant L_{2} .
$$

This assumption is very restrictive, not only mathematically, but also physically: Magnetic heads do not generally satisfy the concavity condition (1.8). Indeed, in order to reduce the effect of air entrainment (which causes a boundary layer for the pressure $p$ near $x=L_{2}$ ), trenches are dug into the head (see [2]) and, of course, $\delta(x)$ is discontinuous at the edges of the trench. But even if a trench is smoothed near the edges so that $\delta$ is a smooth function in the neighborhood of a trench, $\delta^{\prime \prime}(x)$ will change sign across the trench.

For clarity we shall first prove Theorem 1.1, replacing (1.6) by the stronger assumption

$$
\delta \in C^{2}\left[L_{1}, L_{2}\right]
$$

the proof for this special case is given in Sections 2-4. In Section 2 we establish the existence of a solution in the case $\varepsilon=\mu=0$, and in Section 3 we prove that the problem for $\varepsilon=\mu=0$ can be written as a variational inequality. The approach we use to establish these results is entirely different from the approach in [4]; instead of the shooting method used in [4] we use here a method based in sub- and super-solutions. In Section 4 we prove Theorem 1.1 (under the stronger assumption (1.9)) by combining the method used in [4] with the results of Sections 2 and 3. The proof of Theorem 1.1 in the general case is given in Section 5.

\section{THE CASE $\varepsilon=0, \mu=0$}

In the special case $\varepsilon=\mu=0$, the system (1.1)-(1.3) reduces to

$$
\begin{aligned}
& \frac{\partial(p h)}{\partial x}=0, \quad L_{1}<x<L_{2}, \\
& -\frac{\partial^{2} u}{\partial x^{2}}=K(p-1) \chi_{\left[L_{1}, L_{2}\right]}, \quad 0<x<L .
\end{aligned}
$$


Some of the boundary conditions in (1.4), (1.5) need to the dropped, and we take

$$
\begin{gathered}
p\left(L_{1}\right)=1, \\
u(0)=u(L)=0 .
\end{gathered}
$$

From (2.1) we see that $p h=\mathrm{constant}=C$ and, since $p\left(L_{1}\right)=1, C=h\left(L_{1}\right)=$ $u\left(L_{1}\right)-\delta\left(L_{1}\right)$, so that

$$
p(x)=\frac{u\left(L_{1}\right)-\delta\left(L_{1}\right)}{u(x)-\delta(x)}
$$

Hence (2.2) becomes

$$
-\frac{\partial^{2} u}{\partial x^{2}}=K\left(\frac{u\left(L_{1}\right)-\delta\left(L_{1}\right)}{u(x)-\delta(x)}-1\right) \chi_{\left[L_{1}, L_{2}\right]}, \quad 0<x<L .
$$

THEOREM 2.1. There exists a solution $u(x)$ of (2.5), (2.4).

The proof requires several lemmas. Let

$$
A=\left\|\delta^{\prime}\right\|_{L^{\infty}\left(L_{1}, L_{2}\right)}(A>0), \quad B=\sup _{L_{1} \leqslant x \leqslant L_{2}}\left[-\delta^{\prime \prime}(x)\right](B>0) .
$$

For any

$$
\lambda \in I \equiv\left(\delta\left(L_{1}\right), A L_{1}\right]
$$

consider the problem

$$
\begin{gathered}
-\frac{\partial^{2} u}{\partial x^{2}}=K\left(\frac{\lambda-\delta\left(L_{1}\right)}{u(x)-\delta(x)}-1\right) \chi_{\left[L_{1}, L_{2}\right]}, \quad 0<x<L, \\
u(0)=u(L)=0, \\
u(x)-\delta(x)>0 \quad \text { if } L_{1} \leqslant x \leqslant L_{2} .
\end{gathered}
$$

Lemma 2.1. For any $\lambda \in I$ there exists a unique solution $u(x, \lambda)$ of $(2.6)$. Proof. Set

$$
F(u, x)=K\left(\frac{\lambda-\delta\left(L_{1}\right)}{u-\delta(x)}-1\right) \chi_{\left[L_{1}, L_{2}\right]}, \quad u>\delta(x),
$$

and note that

$$
F_{u}<0
$$


Consider the function

$$
\underline{u}(x)=\bar{\delta}(x)+\frac{\lambda-\delta\left(L_{1}\right)}{1+c(\lambda)}
$$

where

$$
\begin{aligned}
& \bar{\delta}(x)= \begin{cases}\delta\left(L_{1}\right)+\delta^{\prime}\left(L_{1}\right)\left(x-L_{1}\right), & 0 \leqslant x<L_{1} \\
\delta(x), & L_{1} \leqslant x \leqslant L_{2} \\
\delta\left(L_{2}\right)+\delta^{\prime}\left(L_{2}\right)\left(x-L_{2}\right), & L_{2}<x \leqslant L,\end{cases} \\
& c(\lambda)=\max \left\{\frac{B}{K}, \frac{\lambda-\delta\left(L_{1}\right)}{-\bar{\delta}(0)}, \frac{\lambda-\delta\left(L_{1}\right)}{-\bar{\delta}(L)}\right\} .
\end{aligned}
$$

Then

$$
\begin{aligned}
-\underline{u}_{x x}-F(\underline{u}(x), x) & \leqslant\left\{B-K\left(\frac{\lambda-\delta\left(L_{1}\right)}{\left(\delta(x)+\frac{\lambda-\delta\left(L_{1}\right)}{1+c(\lambda)}\right)-\delta(x)}-1\right)\right\} \chi_{\left[L_{1}, L_{2}\right]}, \\
& =[B-K c(\lambda)] \chi_{\left[L_{1}, L_{2}\right]}
\end{aligned}
$$

so that, by the choice of $c(\lambda)$,

$$
-\underline{u}_{x x}(x) \leqslant F(\underline{u}(x), x) \text {. }
$$

Furthermore,

$$
\underline{u}(0)=\delta\left(L_{1}\right)-\delta^{\prime}\left(L_{1}\right) L_{1}+\frac{\lambda-\delta\left(L_{1}\right)}{1+c(\lambda)}<0,
$$

and, similarly, $\underline{u}(L)<0$. Thus $\underline{u}$ is a subsolution for the problem (2.6).

The function $\bar{u}(x)=A x$ is a supersolution. Indeed, since $(\bar{u}-\delta)^{\prime}=$ $A-\delta^{\prime} \geqslant 0$,

$$
\bar{u}(x)-\delta(x) \geqslant \bar{u}\left(L_{1}\right)-\delta\left(L_{1}\right)=A L_{1}-\delta\left(L_{1}\right) \geqslant \lambda-\delta\left(L_{1}\right)
$$

if $L_{1} \leqslant x \leqslant L_{2}$, so that

$$
-\bar{u}_{x x}(x)-K\left(\frac{\lambda-\delta\left(L_{1}\right)}{\bar{u}(x)-\delta(x)}-1\right) \chi_{\left[L_{1}, L_{2}\right]} \geqslant-\bar{u}_{x x}(x)=0 .
$$

Furthermore, $\bar{u}(0)=0$ and $\bar{u}(L) \geqslant 0$. 
Introduce the convex set of functions

$$
G=\left\{\tilde{u} \in C^{0}[0, L], \underline{u}(x) \leqslant \tilde{u} \leqslant \bar{u}\right\} .
$$

For any $\tilde{u} \in G$ we consider the problem

$$
\begin{gathered}
-u_{x x}=F(\tilde{u}, x), \quad 0<x<L, \\
u(0)=u(L)=0 .
\end{gathered}
$$

Using (2.7) and the maximum principle (or comparison) we deduce that the solution $u$ satisfies

$$
\underline{u} \leqslant u \leqslant \bar{u}, \quad 0 \leqslant x \leqslant L .
$$

If we define a mapping $T$ by $T(\tilde{u})=u$, then $T$ maps $G$ into itself. It is easily seen that $T$ is continuous and $T(G)$ lies in a compact subset of $G$. Appealing to the Schauder fixed point theorem we conclude that $T$ has a fixed point, which is clearly the solution to (2.6). Finally, if $u_{1}$ is another solution, then the function $w=u-u_{1}$ satisfies

$$
w_{x x}+F_{u} w=0
$$

where $F_{u}$ is evaluated at some intermediate point. Since $F_{u}<0, w \equiv 0$ and thus $u_{1}=u$.

We denote the solution of (2.6) by $u(x, \lambda)$ and introduce the function

$$
f(\lambda)=u\left(L_{1}, \lambda\right), \quad \lambda \in I .
$$

LeMmA 2.2. The function $f$ is continuous.

Proof. If $\lambda_{n} \rightarrow \lambda_{0} \in I$ then any subsequence of $\lambda_{n}$ has a sub-subsequence $\lambda_{n^{\prime}}$ for which $u\left(x, \lambda_{n^{\prime}}\right)$ is uniformly convergent to a function $u_{0}(x)$, and $u_{0}(x)$ is the solution of (2.6) for $\lambda=\lambda_{0}$ (by uniqueness). It follows that

$$
f\left(\lambda_{n}\right)=u\left(L_{1}, \lambda_{n}\right) \rightarrow u\left(L_{1}, \lambda_{0}\right)=f\left(\lambda_{0}\right) .
$$

Proof of Theorem 2.1. We need to show that the mapping $\lambda \rightarrow f(\lambda)$ has a fixed point. If $f\left(\lambda_{1}\right) \geqslant \lambda_{1}$ for some $\lambda_{1} \in I$, then, since $f\left(A L_{1}\right) \leqslant A L_{1}$ and $f(\lambda)$ is continuous, $f$ will have a fixed point in the interval $\left[\lambda_{1}, A L_{1}\right]$. Thus, it remains to prove that the inequality

$$
f(\lambda)<\lambda \quad \text { for all } \quad \lambda \in I
$$


cannot hold. We shall assume that (2.9) holds and proceed to derive the contradiction. To do that we define

$$
\lambda_{n+1}=f\left(\lambda_{n}\right), \quad n>1,
$$

for some $\lambda_{0} \in I$. Then, in view of (2.9), $\lambda_{n} \downarrow \lambda_{*}$, and $\lambda_{*}=\delta\left(L_{1}\right)$ since, otherwise, $f\left(\lambda_{*}\right)=\lambda_{*}$ by Lemma 2.2. Thus

$$
\lambda_{n} \downarrow \delta\left(L_{1}\right) \quad \text { as } n \rightarrow \infty .
$$

Recall that $u_{x x}\left(x, \lambda_{n}\right)=0$ if $0<x<L_{1}$, so that

$$
u_{x}\left(L_{1}, \lambda_{n}\right)=\frac{u\left(L_{1}, \lambda_{n}\right)}{L_{1}}=\frac{\lambda_{n+1}}{L_{1}} \rightarrow \frac{\delta\left(L_{1}\right)}{L_{1}} .
$$

Since, by (1.7),

$$
\delta^{\prime}\left(L_{1}\right)>\frac{\delta\left(L_{1}\right)}{L_{1}}+3 \varepsilon_{0}
$$

for some $\varepsilon_{0}>0$, it follows that

$$
u_{x}\left(L_{1}, \lambda_{n}\right)<\delta^{\prime}\left(L_{1}\right)-2 \varepsilon_{0}
$$

if $n$ is sufficiently large. We also have

$$
u_{x x}\left(L_{1}+0, \lambda_{n}\right)=K\left(1-\frac{\lambda_{n}-\delta\left(L_{1}\right)}{\lambda_{n+1}-\delta\left(L_{1}\right)}\right)<0
$$

since $\lambda_{n+1}=f\left(\lambda_{n}\right)<\lambda_{n}$.

Let

$$
\bar{x}=\max \left\{x \in\left(L_{1}, L_{2}\right] ; \delta^{\prime}\left(L_{1}\right)-\delta^{\prime}\left(x^{\prime}\right) \leqslant \varepsilon_{0}, \text { for all } L_{1} \leqslant x^{\prime} \leqslant x\right\} .
$$

We claim that

$$
u_{x x}\left(x, \lambda_{n}\right)<0 \quad \text { if } \quad L_{1} \leqslant x<\bar{x} .
$$

Indeed, if this is not true then, setting for simplicity $u(x)=u\left(x, \lambda_{n}\right)$, we have, by (2.12),

$$
\begin{aligned}
& u_{x x}(x)<0 \quad \text { if } \quad L_{1} \leqslant x<x^{*}, \\
& u_{x x}\left(x^{*}\right)=0
\end{aligned}
$$


for some $x^{*}=x_{n}^{*} \in\left(L_{1}, \bar{x}\right)$, and then also

$$
u_{x x x}\left(x^{*}\right) \geqslant 0
$$

From the differential equation in (2.6) we get

$$
u_{x x x}(x)=K \frac{\lambda-\delta\left(L_{1}\right)}{(u(x)-\delta(x))^{2}}\left(u_{x}(x)-\delta^{\prime}(x)\right) \quad \text { in }\left(L_{1}, L_{2}\right)
$$

so that, by (2.11),

$$
u_{x x x}\left(L_{1}+0\right)<0
$$

If follows that there exists a point $x^{* *}=x_{n}^{* *}$ in $\left(L_{1}, x^{*}\right]$ such that

$$
\begin{aligned}
u_{x x x}(x)<0 & \text { if } \quad L_{1}<x<x^{* *}, \\
u_{x x x}\left(x^{* *}\right)=0 . &
\end{aligned}
$$

Appealing again to (2.16), we deduce that

$$
u_{x}\left(x^{* *}\right)-\delta^{\prime}\left(x^{* *}\right)=0
$$

and then, by (2.15) and (2.11),

$$
\delta^{\prime}\left(x^{* *}\right)=u_{x}\left(x^{* *}\right)<u_{x}\left(L_{1}\right)<\delta^{\prime}\left(L_{1}\right)-2 \varepsilon_{0},
$$

which is a contradiction to (2.13) since $x^{* *}<\bar{x}$.

From (2.14) and (2.11), (2.13) we deduce that

$$
u_{x}\left(x, \lambda_{n}\right)<u_{x}\left(L_{1}, \lambda_{n}\right)<\delta^{\prime}\left(L_{1}\right)-2 \varepsilon_{0} \leqslant \delta^{\prime}(x)-\varepsilon_{0} \quad \text { if } \quad L_{1}<x \leqslant \bar{x} \text {. }
$$

Hence

$$
u\left(\bar{x}, \lambda_{n}\right)-\delta(\bar{x})<\lambda_{n+1}-\delta\left(L_{1}\right)-\varepsilon_{0}\left(\bar{x}-L_{1}\right)
$$

Recalling (2.10) and the fact that $\bar{x}$ is independent of $n$, we get

$$
u\left(\bar{x}, \lambda_{n}\right)-\delta(\bar{x})<0
$$

if $n$ is sufficiently large, which contradicts the inequality $u\left(x, \lambda_{n}\right)>\delta(x)$ in $\left[L_{1}, L_{2}\right]$. 


\section{VARIATIONAL FORMULATION FOR EQUATIONS (2.5) AND (2.4)}

Theorem 2.1 can be extended to the system

$$
\begin{aligned}
-\frac{\partial^{2} g}{\partial x^{2}} & =K\left(\frac{g\left(L_{1}\right)-\delta\left(L_{1}\right)}{g(x)-\delta(x)}-1\right) \chi_{\left[L_{1}, L_{2}\right]}, \quad 0<x<L, \\
g(0) & =g(L)=-\gamma, \\
g(x)-\delta(x) & >0 \quad \text { if } \quad L_{1} \leqslant x \leqslant L_{2},
\end{aligned}
$$

provided

$$
\max \left\{\delta\left(L_{1}\right)-\delta^{\prime}\left(L_{1}\right) L_{1}, \delta\left(L_{2}\right)-\delta^{\prime}\left(L_{2}\right)\left(L_{2}-L\right)\right\}<-\gamma<0 .
$$

Note that in the construction of the solution to

$$
\begin{aligned}
-\frac{\partial^{2} g}{\partial x^{2}} & =K\left(\frac{\lambda-\delta\left(L_{1}\right)}{g(x)-\delta(x)}-1\right) \chi_{\left[L_{1}, L_{2}\right]}, \quad 0<x<L, \\
g(0) & =g(L)=-\gamma, \\
g(x)-\delta(x) & >0 \quad \text { if } \quad L_{1} \leqslant x \leqslant L_{2},
\end{aligned}
$$

we use the same sub- and super-solutions $\underline{u}(x)$ and $\bar{u}(x)$, respectively, as before. We shall denote the solution of (3.3) by $g(x, \lambda, \gamma)$.

Set

$$
F(u, x, \lambda)=K\left(\frac{\lambda-\delta\left(L_{1}\right)}{u-\delta(x)}-1\right) \chi_{\left[L_{1}, L_{2}\right]}, \quad u>\delta(x) .
$$

Clearly

$$
F_{u}<0, \quad F_{\lambda}>0
$$

By the strong maximum principle we then get

$g\left(x, \lambda_{1}, \gamma_{1}\right)>g\left(x, \lambda_{2}, \gamma_{2}\right) \quad$ in $\quad(0, L) \quad$ if $\quad \lambda_{1} \geqslant \lambda_{2}, \quad 0 \leqslant \gamma_{1}<\gamma_{2}$.

LEMMA 3.1. Let $g(x)$ be any solution of (3.1). Then there exists a solution $u(x)$ of $(2.5),(2.4)$ such that

$$
u(x)>g(x) \quad \text { if } \quad 0<x<L,
$$


and, consequently,

$$
u(x) \geqslant g(x)+\varepsilon_{0}, \quad \text { if } \quad L_{1} \leqslant x \leqslant L_{2},
$$

for some $\varepsilon_{0}>0$.

Proof. By (3.4) and the maximum principle,

$$
u(x, \lambda)>g(x, \lambda, \gamma) \quad \text { if } \quad 0<x<L .
$$

Take $\lambda=\lambda_{0}$ such that $g\left(x, \lambda_{0}, \gamma\right)$ is the solution $g(x)$. Then

$$
u\left(L_{1}, \lambda_{0}\right)>g\left(L_{1}, \lambda_{0}, \gamma\right)=\lambda_{0}
$$

so that $f\left(\lambda_{0}\right)>\lambda_{0}$. It follows that there is a fixed point $\lambda_{*}$ of the mapping $\lambda \rightarrow f(\lambda)$ in the interval $\left(\lambda_{0}, A L_{1}\right]$. The function $u(x)=u\left(x, \lambda_{*}\right)$ is the solution of (2.5), (2.4) and by (3.4), (3.7),

$$
u(x)=u\left(x, \lambda_{*}\right) \geqslant u\left(x, \lambda_{0}\right)>g(x) \quad \text { in } \quad(0, L) .
$$

Introduce the function

$$
\psi(s)= \begin{cases}s & s<1+A L_{1} \\ 1+A L_{1} & s \geqslant 1+A L_{1} .\end{cases}
$$

Take any solution $g(x)$ of (3.1) and consider the variational inequality

$$
\begin{gathered}
-\frac{\partial^{2} u}{\partial x^{2}} \geqslant K\left(\frac{\psi\left(u\left(L_{1}\right)\right)-\delta\left(L_{1}\right)}{u(x)-\delta(x)}-1\right) \chi_{\left[L_{1}, L_{2}\right]} \\
u(x) \geqslant g(x), \\
(u-g)\left[\frac{\partial^{2} u}{\partial x^{2}}+K\left(\frac{\psi\left(u\left(L_{1}\right)\right)-\delta\left(L_{1}\right)}{u(x)-\delta(x)}-1\right) \chi_{\left[L_{1}, L_{2}\right]}\right]=0 \quad \text { in }[0, L], \\
u(0)=u(L)=0 .
\end{gathered}
$$

The truncation $\psi$ is introduced for a technical reason, so that we can carry out the proof of Lemma 4.1 in Section 4; see also [4].

Note that the solution $u$ of (2.5), (2.4) established in Lemma 3.1 satisfies the variational inequality (3.8) since $\psi\left(u\left(L_{1}\right)\right)=u\left(L_{1}\right)$ (as $\left.u\left(L_{1}\right) \leqslant A L_{1}\right)$. We now prove the converse:

THEOREM 3.1. Any solution $u(x)$ of the variational inequality (3.8) is a solution of (2.5), (2.4), and it satisfies the inequalities (3.5), (3.6). 
Proof. Since $u\left(L_{1}\right) \geqslant g\left(L_{1}\right), \psi(s)$ is monotone increasing in $s$, and $g\left(L_{1}\right) \leqslant A L_{1}$,

$$
\psi\left(u\left(L_{1}\right)\right)-\delta\left(L_{1}\right) \geqslant \psi\left(g\left(L_{1}\right)\right)-\delta\left(L_{1}\right)=g\left(L_{1}\right)-\delta\left(L_{1}\right) .
$$

It follows that

$$
-\frac{\partial^{2} u}{\partial x^{2}} \geqslant K\left(\frac{g\left(L_{1}\right)-\delta\left(L_{1}\right)}{u(x)-\delta(x)}-1\right) \chi_{\left[L_{1}, L_{2}\right]} .
$$

Since, furthermore,

$$
u(0)=u(L)>-\gamma=g(0)=g(L),
$$

the strong maximum principle yields the inequalities (3.5), (3.6). It follows that

$$
-\frac{\partial^{2} u}{\partial x^{2}}=K\left(\frac{\psi\left(u\left(L_{1}\right)\right)-\delta\left(L_{1}\right)}{u(x)-\delta(x)}-1\right) \chi_{\left[L_{1}, L_{2}\right]} .
$$

From the inequality $\psi(s) \leqslant s$ we then have that

$$
-\frac{\partial^{2} u}{\partial x^{2}} \leqslant K\left(\frac{u\left(L_{1}\right)-\delta\left(L_{1}\right)}{u(x)-\delta(x)}-1\right) \chi_{\left[L_{1}, L_{2}\right]},
$$

and, since $\bar{u}(x)=A x$ is a supersolution, we get, by comparison, $u(x) \leqslant A x$. This implies that $\psi\left(u\left(L_{1}\right)\right)=u\left(L_{1}\right)$, so that (3.9) reduces to (2.5). Thus $u(x)$ is a solution of (2.5), (2.4) satisfying (3.5), (3.6).

\section{PROOF OF THEOREM 1.1 (SPECIAL CASE)}

Consider the system

$$
\begin{gathered}
\frac{\partial(p h)}{\partial x}-\varepsilon \frac{\partial}{\partial x}\left(\alpha h^{2} \frac{\partial p}{\partial x}+\beta h^{3} p \frac{\partial p}{\partial x}\right)=0, \quad L_{1}<x<L_{2}, \\
-\frac{\partial^{2} u}{\partial x^{2}}+\mu \frac{\partial^{4} u}{\partial x^{4}} \geqslant K(p-1) \chi_{\left[L_{1}, L_{2}\right]}, \quad u(x) \geqslant g(x), \\
(u-g)\left[-\frac{\partial^{2} u}{\partial x^{2}}+\mu \frac{\partial^{4} u}{\partial x^{4}}-K(p-1) \chi_{\left[L_{1}, L_{2}\right]}\right]=0 \quad \text { in } \quad[0, L], \\
h(x)=u(x)-\delta(x), \quad L_{1} \leqslant x \leqslant L_{2},
\end{gathered}
$$


with the boundary conditions

$$
\begin{gathered}
p\left(L_{1}\right)=p\left(L_{2}\right)=\frac{\psi\left(h\left(L_{1}\right)\right)}{h\left(L_{1}\right)} \\
u=u_{x}=0 \quad \text { at } \quad x=0 \quad \text { and } \quad x=L .
\end{gathered}
$$

Let

$$
G=\left\{p \in C^{0}\left[L_{1}, L_{2}\right], 0 \leqslant p \leqslant 1+\frac{1+A L_{1}}{l}\right\},
$$

where

$$
l=\inf _{L_{1} \leqslant x \leqslant L_{2}}[g(x)-\delta(x)]>0 .
$$

As in [4] one shows that for any $p \in G$ there exists a unique solution $u$ of (4.2), (4.5) and

$$
\left|u_{x}\right| \leqslant C
$$

where $C$ is a constant independent of $p$.

Set $h=u-\delta$ and denote by $\hat{p}(x)$ the solution of (4.1), (4.4). It is obtained by solving the initial value problem

$$
\begin{aligned}
\hat{p} h-\varepsilon\left(\alpha h^{2} \frac{\partial \hat{p}}{\partial x}+\beta h^{3} \hat{p} \frac{\partial \hat{p}}{\partial x}\right) & =\eta, \quad L_{1}<x<L_{2}, \\
\hat{p}\left(L_{1}\right) & =\frac{\psi\left(h\left(L_{1}\right)\right)}{h\left(L_{1}\right)},
\end{aligned}
$$

for any constant $\eta \geqslant 0$, and then choosing $\eta$ in a unique way such that

$$
\hat{p}\left(L_{2}\right)=\frac{\psi\left(h\left(L_{1}\right)\right)}{h\left(L_{1}\right)}
$$

for details see [4].

We define the mapping $S$ by

$$
S(p)=\hat{p}
$$

and want to show that $S$ has a fixed point in $\mathrm{G}$. 
Lemma 4.1. If $\varepsilon$ is small enough then $S$ maps $G$ into itself and the parameter $\eta$ determined by (4.7), (4.8) satisfies

$$
\left|\eta-\psi\left(h\left(L_{1}\right)\right)\right| \leqslant C \varepsilon
$$

where $C$ is a constant independent of $\varepsilon$.

Proof. We introduce the operator

$$
\mathscr{L}(w) \equiv \frac{\partial(w h)}{\partial x}-\varepsilon \frac{\partial}{\partial x}\left(\alpha h^{2} \frac{\partial w}{\partial x}+\beta h^{3} w \frac{\partial w}{\partial x}\right), \quad L_{1}<x<L_{2},
$$

and consider the function

$$
v(x)=\frac{\psi\left(h\left(L_{1}\right)\right)}{h\left(L_{1}\right)}-\frac{C}{l}\left(x-L_{1}\right)-v, \quad L_{1} \leqslant x \leqslant L_{2}
$$

for $C$ positive and large and $v$ positive and arbitrarily small. Denote by $\hat{x}$ the point where $v(\hat{x})=0$. Then, as in [4],

$$
\mathscr{L}(v)<0 \quad \text { in } \quad\left[L_{1}, \hat{x}\right]
$$

if $\varepsilon$ is small enough. A comparison argument used in [4] then shows that

$$
\hat{p}(x) \geqslant v(x) \quad \text { in } \quad\left[L_{1}, \hat{x}\right] .
$$

Taking $v \downarrow 0$ we deduce that

$$
p_{x}\left(L_{1}\right)>-\frac{C}{l}
$$

and, then, by (4.7),

$$
\eta-\psi\left(h\left(L_{1}\right)\right) \leqslant C \varepsilon
$$

The complementary inequality

$$
\eta-\psi\left(h\left(L_{1}\right)\right) \geqslant-C \varepsilon .
$$

is derived in the same way by working with the function

$$
\frac{\psi\left(h\left(L_{1}\right)\right)}{h\left(L_{1}\right)}+\frac{C}{l}\left(x-L_{1}\right)+v .
$$

Since

$$
\psi\left(h\left(L_{1}\right)\right) \leqslant 1+A L_{1}
$$


it follows from (4.9) that

$$
\eta \leqslant 1+A L_{1}+C \varepsilon
$$

(This is where we need the truncation $\psi$, since we do not have a uniform bound on $h\left(L_{1}\right)$.) But, then, from (4.7) we conclude that

$$
\hat{p} \leqslant 1+\frac{1+A L_{1}}{l},
$$

if $\varepsilon$ is sufficient small.

From (4.7) we also deduce that $\hat{p}$ cannot take the negative minimum, and thus $\hat{p} \in G$, and so $S$ maps $G$ into itself.

Proof of Theorem 1.1. By Lemma 4.1, $S$ maps $G$ into itself. It is also easy to show that $S$ is continuous and that $S(G)$ is contained in a compact subset of $G$. Hence, by Schauder's fixed point theorem, $S$ has a fixed point in $G$, which is solution $(u, p)$ of the system (4.1)-(4.5). If we can prove that

$$
\begin{gathered}
u(x)>g(x) \quad \text { in }(0, L), \\
u\left(L_{1}\right)<1+A L_{1},
\end{gathered}
$$

for $0<\varepsilon<\varepsilon_{*}$ and $0<\mu<\mu_{*}$, then $(u, p)$ and $h$ form a solution of (1.1) $-(1.5)$ as asserted in Theorem 1.1.

But if either (4.10) or (4.11) is not satisfied, then, by taking the sequences $\varepsilon_{j} \downarrow 0, \mu_{j} \downarrow 0$ as in [4], we obtain the limit functions $\left(u_{0}, p_{0}, h_{0}\right)$ with

$$
p_{0}(x) h_{0}=\frac{\psi\left(h_{0}\left(L_{1}\right)\right)}{h_{0}\left(L_{1}\right)} \quad(\operatorname{using}(4.9))
$$

and $u_{0}(x)$ satisfying the variational inequality (3.8). By Theorem 3.1,

$$
u_{0}(x) \geqslant g(x)+\varepsilon_{0} \quad \text { for } \quad L_{1} \leqslant x \leqslant L_{2}
$$

and clearly also

$$
u_{0}(x) \leqslant A L_{1} .
$$

But then, for the solution of (4.1)-(4.5), with $\varepsilon=\varepsilon_{j}, \mu=\mu_{j}$ small,

$$
u(x) \geqslant g(x)+\frac{1}{2} \varepsilon_{0} \quad \text { for } \quad L_{1} \leqslant x \leqslant L_{2},
$$

and $u\left(L_{1}\right)<1+A L_{1}$. Since (4.12) implies (4.10), we get a contradiction to both inequalities, (4.10) and (4.11). 


\section{PROOF OF THEOREM 1.1}

We introduce the function

$$
\hat{\delta}(x)=\delta\left(L_{1}\right)+\left(\left(\xi_{1}-L_{1}\right)\left\|\delta^{\prime}\right\|_{L^{\infty}\left[L_{1}, \xi_{1}\right]}+\|\delta\|_{L^{\infty}\left[\xi_{1}, L_{2}\right]}\right) \frac{x-L_{1}}{\xi_{1}-L_{1}}
$$

for $0 \leqslant x \leqslant L$ and note that

$$
\delta(x) \leqslant \hat{\delta}(x) \quad \text { if } \quad L_{1} \leqslant x \leqslant L_{2}
$$

We first prove Theorem 2.1 (under the assumption (1.6)). Since $-\delta^{\prime \prime}$ is not bounded from above (if $s \geqslant 1$ in (1.6)), we cannot construct a subsolution as before. We therefore first approximate $\delta(x)$ by functions $\delta_{n}$ in $C^{2}\left[L_{1}, L_{2}\right]$ such that

$$
\begin{gathered}
\int_{L_{1}}^{L_{2}}\left|\delta_{n}(x)-\delta(x)\right|^{p} d x \rightarrow 0 \quad \text { for any } 1<p<\infty, \\
\delta_{n}\left(L_{1}\right)=\delta\left(L_{1}\right), \\
\sup _{\substack{L_{1} \leqslant x \leqslant L_{1}+\eta_{0} \\
L_{2}-\eta_{0} \leqslant x \leqslant L_{2}}}\left|\delta_{n}^{\prime}(x)-\delta^{\prime}(x)\right| \rightarrow 0
\end{gathered}
$$

for some small $\eta_{0}>0$,

$$
\delta_{n}^{\prime}(x) \rightarrow \delta^{\prime}(x) \quad \text { for all } \quad x \neq \xi_{i}
$$

as $n \rightarrow \infty$, and

$$
\delta_{n}(x) \leqslant \hat{\delta}(x)
$$

Consider the problem

$$
\begin{aligned}
& -v^{\prime \prime}=K\left(\frac{\lambda-\delta\left(L_{1}\right)}{v(x)-\delta_{n}(x)}-1\right) \chi_{\left[L_{1}, L_{2}\right]}, \quad 0<x<L, \\
& v(0)=v(L)=0 .
\end{aligned}
$$

Lemma 5.1. The function $\bar{u}(x)=\hat{\delta}(x)-\hat{\delta}(0)$ is a supersolution of $(5.8)$ provided $\lambda \leqslant \bar{u}\left(L_{1}\right)$.

Proof. Clearly

$$
\bar{u}(x)-\delta_{n}(x)=\hat{\delta}(x)-\delta_{n}(x)-\hat{\delta}(0) \geqslant-\hat{\delta}(0),
$$


by (5.7). Also,

$$
\hat{\delta}\left(L_{1}\right)-\delta(0)=\bar{u}\left(L_{1}\right) \geqslant \lambda
$$

so that

$$
-\hat{\delta}(0) \geqslant \lambda-\hat{\delta}\left(L_{1}\right)=\lambda-\delta\left(L_{1}\right)
$$

Hence

$$
\bar{u}(x)-\delta_{n}(x) \geqslant \lambda-\delta\left(L_{1}\right)
$$

and, consequently,

$$
-\bar{u}_{x x}(x)-K\left(\frac{\lambda-\delta\left(L_{1}\right)}{\bar{u}(x)-\delta_{n}(x)}-1\right) \chi_{\left[L_{1}, L_{2}\right]} \geqslant-\bar{u}_{x x}(x)=0 .
$$

Since also $\bar{u}(0)=0$ and $\bar{u}(L)>0$, the lemma follows.

It seems difficult to construct a subsolution to the $u_{n}$ which is independent of $n$. But we can nevertheless apply Theorem 2.1 (for the case where (1.9) is satisfied) to deduce that there exists a solution $u_{n}(x)$ of

$$
\begin{aligned}
-u_{n}^{\prime \prime}(x) & =K\left(\frac{u_{n}\left(L_{1}\right)-\delta\left(L_{1}\right)}{u_{n}(x)-\delta_{n}(x)}-1\right) \chi_{\left[L_{1}, L_{2}\right]}, \quad 0<x<L, \\
u_{n}(0) & =u_{n}(L)=0
\end{aligned}
$$

and

$$
\delta_{n}(x)<u_{n}(x) \leqslant \bar{u}(x), \quad 0<x<L .
$$

We may assume that

$$
u_{n}\left(L_{1}\right) \rightarrow \lambda_{*}
$$

for some $\delta\left(L_{1}\right) \leqslant \lambda_{*} \leqslant \bar{u}\left(L_{1}\right)$.

LEMma 5.2. There holds

$$
\lambda_{*}>\delta\left(L_{1}\right)
$$

Proof. Suppose $\lambda_{*}=\delta\left(L_{1}\right)$. Then, for any small $\varepsilon_{0}>0$,

$$
u_{n}\left(L_{1}\right)<\delta\left(L_{1}\right)+L_{1} \varepsilon_{0} \quad \text { if } n \geqslant n_{0}\left(\varepsilon_{0}\right)
$$


and

$$
u_{n}^{\prime}\left(L_{1}\right)=\frac{u_{n}\left(L_{1}\right)}{L_{1}}<\frac{\delta\left(L_{1}\right)}{L_{1}}+\varepsilon_{0}<\delta^{\prime}\left(L_{1}\right)-2 \varepsilon_{0}<\delta_{n}^{\prime}\left(L_{1}\right)-\varepsilon_{0}
$$

if $\varepsilon_{0}$ is small enough, by (1.7), (5.5).

We can now argue as in the proof of (2.14) (with $u=u_{n}, \delta=\delta_{n}$, and $\left.\lambda_{n}=u_{n}\left(L_{1}\right)\right)$ to deduce that

$$
u_{n}^{\prime \prime}(x)<0 \quad \text { if } \quad L_{1} \leqslant x \leqslant \bar{x}
$$

where $\bar{x}$ is such that $\bar{x} \leqslant \eta_{0}$ and $\delta_{n}^{\prime}\left(L_{1}\right)-\delta_{n}^{\prime}(x)<\frac{1}{2} \varepsilon_{0}$ for all $L_{1} \leqslant x \leqslant \bar{x}$, $n \geqslant n_{0}\left(\varepsilon_{0}\right)$. Using also (5.13), we deduce that

$$
u_{n}^{\prime}(x)<\delta_{n}^{\prime}(x)-\frac{\varepsilon_{0}}{2} \quad \text { if } \quad L_{1} \leqslant x \leqslant \bar{x}
$$

It follows that

$$
u_{n}(\bar{x})-\delta_{n}(\bar{x})<\left(u_{n}\left(L_{1}\right)-\delta_{n}\left(L_{1}\right)\right)-\frac{\varepsilon_{0}}{2}\left(\bar{x}-L_{1}\right)
$$

Hence $u_{n}(\bar{x})-\delta_{n}(\bar{x})<0$ if $n$ is sufficiently large, a contradiction.

Since $u_{n}^{\prime \prime}(x)=0$ and $0<u_{n}(x) \leqslant \bar{u}(x)$ for $0<x<L_{1}$ and $L_{2}<x<L$, we have

$$
0 \leqslant u_{n}^{\prime}(0) \leqslant \bar{u}^{\prime}(0), \quad \text { and } \quad 0>u_{n}^{\prime}(L)=\frac{u_{n}\left(L_{2}\right)}{L_{2}-L} \geqslant \frac{\bar{u}\left(L_{2}\right)}{L_{2}-L}
$$

Integrating the differential equation in (5.9) and using (5.14) we obtain the inequality

$$
\int_{L_{1}}^{L_{2}} \frac{u_{n}\left(L_{1}\right)-\delta\left(L_{1}\right)}{u_{n}(x)-\delta_{n}(x)} d x \leqslant C .
$$

In view of (5.10) and (5.11), (5.12), the last inequality implies that

$$
\int_{L_{1}}^{L_{2}} \frac{d x}{u_{n}(x)-\delta_{n}(x)} \leqslant C
$$

Integrating (5.9) and using (5.15), (5.14) we see that

$$
\left|u_{n}^{\prime}(x)\right| \leqslant C, \quad 0<x<L .
$$


We may then assume that

$$
\begin{gathered}
u_{n}(x) \rightarrow u(x) \quad \text { uniformly in } x \in[0, L], \\
\left|u^{\prime}(x)\right| \leqslant C \quad \text { a.e. }
\end{gathered}
$$

We next deduce, by Fatou's Lemma and (5.15), that

$$
\int_{L_{1}}^{L_{2}} \frac{d x}{u(x)-\delta(x)} \leqslant C
$$

LEMMA 5.3. There holds:

$$
u(x)-\delta(x)>0 \quad \text { if } \quad L_{1} \leqslant x \leqslant L_{2} .
$$

Proof. Suppose $u\left(x_{0}\right)-\delta\left(x_{0}\right)=0$ for some $x_{0} \in\left[L_{1}, L_{2}\right]$. Then, by the Mean Value Theorem,

$$
|u(x)-\delta(x)| \leqslant C\left|x-x_{0}\right|
$$

in an interval with endpoint at $x_{0}$, and this contradicts the estimate (5.18).

Having proved Lemmas 5.2 and 5.3, we can now pass to the limit in (5.9) and conclude that

$$
-u^{\prime \prime}=K\left(\frac{u\left(L_{1}\right)-\delta\left(L_{1}\right)}{u(x)-\delta(x)}-1\right) \chi_{\left[L_{1}, L_{2}\right]}
$$

for $0<x<L, x \neq \xi_{i}$; this completes the proof of Theorem 2.1. Note that $u(x)$ is continuously differentiable in $0 \leqslant x \leqslant L$ and $u^{\prime \prime}(x)$ is piecewise continuous with jump discontinuities at $L_{1}, \xi_{1}, \xi_{2}, \ldots, \xi_{s}, \xi_{s+1}$.

In the same way we can proceed to construct a solution $g$ to (3.1).

We next claim that Lemma 3.1 extends to the present case. Indeed, since both $u(x)$ and $g(x)$ are larger than $\delta(x)$, and $u^{\prime \prime}(x)$ and $g^{\prime \prime}(x)$ are piecewise continuous, the maximum principle can be applied to deduce (3.7).

The proofs of Theorem 3.1 and Theorem 1.1 can now proceed exactly as before.

Remark. As in [4], the solutions asserted in Theorem 1.1 exhibit a boundary layer behavior at $x=L_{2}$.

\section{ACKNOWLEDGMENT}

The first author was partially supported by National Science Foundation Grant DMS 9703842. 


\section{REFERENCES}

1. B. Bhushan, "Tribology and Mechanics of Magnetic Storage Devices," Springer-Verlag, New York, 1990.

2. B. Bhushan, "Mechanics and Reliability of Flexible Magnetic Media," Springer-Verlag, New York, 1992.

3. A. Friedman, "Mathematics in Industrial Problems," Part 7, IMA, Vol.67, SpringerVerlag, New York, 1994.

4. A. Friedman and B. Hu, Head-media interaction in magnetic recording, Arch. Rational Mech. Anal. 140 (1997), 79-101. 